\title{
Ultrastructural Modifications of the Mouse Uterus upon Biogenic Amine Stimulation
}

\author{
S.M.B. Obayomi ${ }^{1}$, S. Peck ${ }^{1}$ and D.P. Baluch ${ }^{1 *}$ \\ 1. School of Life Sciences, Arizona State University, Tempe, Arizona, USA. \\ * Corresponding author: page.baluch@asu.edu
}

Pregnancy and childbirth occur naturally, but still little is known about the signaling mechanisms that induce contractions. Worldwide, premature labor occurs in $12 \%$ of all pregnancies and results in 15 million babies being born preterm [1]. Although the cause of preterm labor can vary, understanding alternative signaling pathways that affect muscle contraction could provide additional treatment options in stopping premature labor.

The uterus is composed of smooth muscle that is innervated with a plexus of nerves that cover the muscle fibers. The plexus contains neurons that have swollen areas called varicosities which contain neurotransmitters. Tyramine and dopamine are both modulators of smooth muscle activity and are found around the smooth muscle of the mouse uterus. Dopamine is a documented neurotransmitter that inhibits smooth muscle contractions while a recent study has found that tyramine, and its specific receptor TAAR1 (trace amine associated receptor 1), are localized within the uterine plexus and can induce uterine contractions [2]. Although dopamine has greater affinity to the vesicular monoamine transporter (VMAT), both modulators can be taken up into synaptic vesicles [3]. It has also been documented that high levels tyramine can displace dopamine, norepinephrine and epinephrine from pre-synaptic vesicles. Identifying and understanding the dynamics of synaptic transmission in the uterus will help uncover the roles that these biogenic amines play in contraction and possibly provide insight to potential treatments that could be used to stop premature labor.

Using both light and electron microscopy, mouse uterine tissue was imaged to determine morphological changes that were induced by treating them with physiological concentrations of either dopamine or tyramine and to correlate localization of these modulators within varicosities at the plexus (not shown). This protocol was approved through the IACUC protocol 15-1388T at Arizona State University (ASU). The tissues were obtained from both a C57BL/6J wild type and a GFP LifeAct transgenic mouse line [4]. Protocols for inducing pseudo-pregnancy, procurement and preparing tissue for immunohistochemistry are detailed in a previous publication [5]. Specific antibody used includes anti-TAAR1 (Santa Cruz Biotechnology). Secondary labeling included Alexa 568 (ThermoFisher). Images were acquired in the core labs housed within the School of Life Sciences at ASU. The instruments used included a Leica SP8 confocal, a JEO1 JSM 6300 SEM and a Philips CM12 TEM system. Results of this project found that dopamine and tyramine treated uterine tissue generated changes in the apical plasma membrane including the height and concentration of peripheral microvilli, increased varicosity swelling and synaptic vesicle cycling in addition to increased mitochondria density and localization [6].

\section{References:}

[1] C Ferre et al., MMWR 65 (2016), p. 1181.

[2] SMB Obayomi et al., Microscopy and Microanalysis 23(S1) (2017), p. 1176.

[3] D Downing et al., in "Advancing Medicine with Food and Nutrients", ed. I Kohlstadt, (CRC Press, 
New York) p. 511.

[4] J Riedl et.al., Nat Methods 5 (2008), p. 605.

[5] DP Baluch and DG Capco, Dev. Biol. 317 (2008), p. 46.

[6] We acknowledge the Graduate and Professional Student Association at ASU for their generous graduate research award funding.


Figure 1. Different Microscope Techniques to Visualize Uterine Smooth Muscle. A confocal image of a mouse uterus cross section shows muscle and vasculature labeled with GFP Lifeact-conjugated actin (green) and the TAAR1 tyramine receptor covering the muscle within the plexus (blue, Fig. A). In Fig. B, a scanning electron micrograph shows a cross section of the uterus where the same lateral and circular smooth muscle fibers can be seen as the fluorescent labeled image in Fig. A. Transmission electron microscopy was used to visualize the varicosities containing synaptic vesicles where the biogenic amines are stored.


Figure 2. TEM Images of the Mouse Uterine Plexus. Within the plexus of untreated mouse uterus, varicosities have a small amount of synaptic vesicles (Fig. A). The number of synaptic vesicles increased in both dopamine (Fig. B) and tyramine (Fig. C) treated tissues as well as an increase in mitochondrial recruitment. 